\title{
UM CIRCUITO DE AUXÍLIO À COMUTAÇÃO QUASI-ZVS APLICADO A CONVERSORES FORWARD COM DOIS INTERRUPTORES
}

\author{
José Renes Pinheiro, José Eduardo Baggio, Luciano Schuch \\ UFSM/CT/DELC/NUPEDEE \\ CEP: $97105-900$ - Santa Maria, RS - Brasil \\ renes@ctlab.ufsm.br,baggio@nupedee.ufsm.br, lschuch@sm.conex.com.br
}

\begin{abstract}
Resumo - Este artigo apresenta um circuito de auxílio à comutação (CAC) aplicado a conversores forward com dois interruptores, operando com comutação do tipo quasi-ZVS, (Quasi Zero Voltage Switching) nos interruptores principais. O CAC utilizado é composto por um indutor ressonante de pequeno volume, um diodo e um interruptor que comuta sob corrente nula. Não é necessário o uso de um terceiro enrolamento no transformador nem fontes de tensão auxiliares para realizar a comutação. A implementação é possível através do uso de um comando apropriado, diferente daquele convencionalmente utilizado em conversores forward com dois interruptores. $\mathbf{E}$ apresentada uma completa análise das etapas de operação bem como os procedimentos de projcto para a correta opcração do conversor. Resultados experimentais foram obtidos de um protótipo de $250 \mathrm{~W} / 48 \mathrm{~V}$ @ $100 \mathrm{kHz}$, provando a viabilidade deste CAC aplicado a conversores forward com dois interruptores. Concomitantemente, um novo conceito de comutação é introduzido, onde os interruptores principais comutam de forma quasi-ZVS.
\end{abstract}

Abstract - This paper presents an active auxiliary circuit (AAC) applied to forward converters with two switches which performs a quasi-ZVS commutation at main switches. The AAC is composed by one resonant inductor of small volume, one diode and one switch that commutates at ZCS (Zero Current Switching). It is not necessary the use of a third winding in the transformer neither auxiliary voltage sources to achieve soft switching. The implementation is possible through the use of an appropriate command, different than it is used conventionally in two switches forward converters. Complete analysis of the operation stages, as well as the design procedures for the correct operation of the converter are presented. Experimental results obtained from a prototype of $250 \mathrm{~W} / 48 \mathrm{~V} @ 100 \mathrm{kHz}$ prove the feasibility of this AAC applied to two switches forward converters. Furthermore, a new concept of switching is introduced, where the main switches commutate at quasiZVS.

\section{INTRODUCุ̃̃o}

Com o avanço da ciência, muitos esforços vem sendo dispensados no intuito de se obter melhor performance e redução de volume dos conversores de potência, uma vez que estes estão presentes na maioria dos aparelhos eletrônicos. Nas ultimas décadas a elevação da freqüência de comutação das dezenas de $\mathrm{kHz}$ às centenas de $\mathrm{kHz}$ permitiua diminuição do volume dos componentes magnéticos e dos capacitores presentes no circuito, resultando em uma diminuição dos volumes dos conversores. Entretanto, com o aumento da frequiência, as perdas de comutação tornam-se bastante expressivas, reduzindo a eficiência e aumentando, conseqüentemente, o volume dos dissipadores. Através de técnicas de comutação suave com comutação sob tensão nula e/ou corrente nula, as perdas de comutação são reduzidas, aumentando a eficiência e reduzindo o volume dos dissipadores dos conversores.

Um cuidado que se deve ter ao se trabalhar com o conversor forward é o de proporcionar a desmagnetização do núcleo do transformador. Comumente, a desmagnetização é feita com o uso de um terceiro enrolamento no transformador. Já no conversor forward com dois interruptores, a desmagnetização é obtida sem a necessidade de um terceiro enrolamento, limitando-se a razão cíclica máxima em $50 \%$.

Em [1 1 e 2] são apresentados circuitos snubber não dissipativo onde os interruptores principais entram em condução sob condição ZCS e são bloqueados sob ZVS. Em [1] existe a desvantagem do indutor ressonante ser colocado em série com a carga, fazendo com que este conduza a corrente de carga, aumentando o volume e o custo deste elemento. Este inconveniente é contornado em [2], onde o snubber é colocado em paralelo com a carga. Entretanto na implementação destes snubber são utilizados MOSFETs, os quais apresentam perdas capacitivas ao entrarem em condução de forma ZCS.

Fazendo uso de um transformador com três enrolamentos, em [3] uma fonte auxiliar é introduzida com o terceiro enrolamento, que juntamente com um circuito ressonante LC e um interruptor auxiliar, permitem a comutação sob tensão nula (ZVS) para os interruptores principais. O terceiro enrolamento do transformador é eliminado em [4], e somente a indutância de magnetização do transformador é utilizada na obtenção da fonte auxiliar para excitação do circuito ressonante.

Em [5] é apresentado um circuito que opera com dois conversores forward acoplados magneticamente, através de um transformador de três enrolamentos, onde a transferência de potência é controlada por deslocamento de fase. Este mesmo circuito apresenta também a vantagem de realizar comutação suave nos interruptores principais.

Em [6] é apresentado um circuito auxiliar composto por um interruptor e um indutor ressonante aplicado a u conversor em ponte completa. Um circuito similar pode ser 
utilizado em conversores forward com dois interruptores, modificando-se o comando dos interruptores principais de forma apropriada.

Este trabalho apresenta um CAC aplicado a conversores forward com dois interruptores. Nesta topologia, o conversor opera com comutação suave nos interruptores principais (entrada em condução Quasi-ZVS e bloqueio ZVS), e comutação suave do tipo ZCS no interruptor do circuito auxiliar.

\section{CirCuito AuXiliar Para UTILIZAÇÃo EM CONVERSORES FORWARD COM DOIS INTERRUPTORES}

A topologia proposta, idealizada, é apresentada na Fig. 1, sendo esta composta por dois interruptores principais $S_{1}$ e $S_{2}$ (MOSFETs), dois diodos principais $\mathrm{D}_{\mathrm{l}}$ e $\mathrm{D}_{2}$, um transformador $\left(1,\left(\mathrm{~N}_{1}, \mathrm{~N}_{2}\right)\right)$, dois diodos retificadores $\left(\mathrm{D}_{3}\right.$, $\left.\mathrm{D}_{4}\right)$ e dois capacitores $\left(\mathrm{C}_{1}\right.$ e $\left.\mathrm{C}_{2}\right)$. A indutância de dispersão do transformador é representada pelo indutor $\mathrm{L}_{\mathrm{d}}$. $\mathrm{O}$ circuito auxiliar possui um indutor ressonante $L_{r}$, um interruptor $S_{a 1}$ (IGBT) e um diodo $\mathrm{D}_{\mathrm{a}}$. O filtro de saída é composto de $\mathrm{u}$ indutor $\mathrm{L}_{\mathrm{f}}$ e um capacitor $\mathrm{C}_{\mathrm{f}}$, sendo a carga representada por $\mathrm{R}_{0}$.

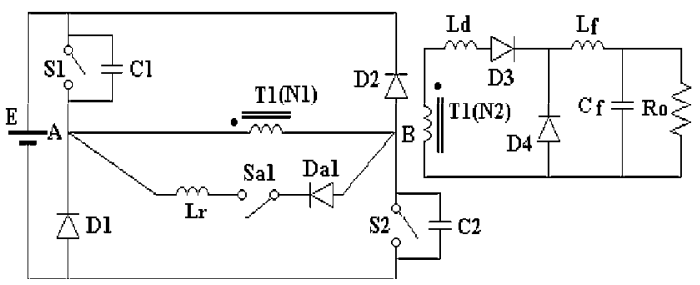

Figura 1 - Topologia proposta, idealizada.

Para a adequada operação do circuito auxiliar, é necessário que os interruptores operem conforme lógica de comando apresentada na Fig. $2 b$.

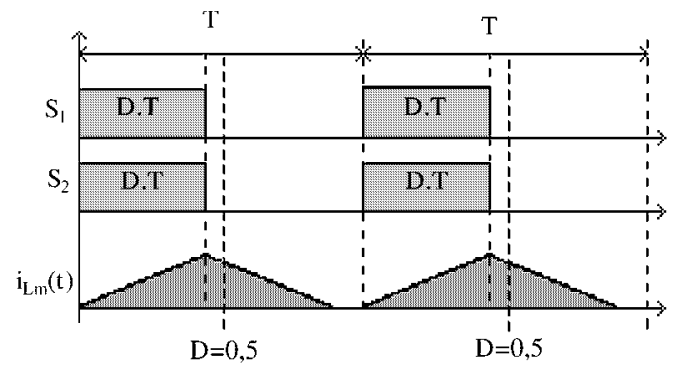

(a)

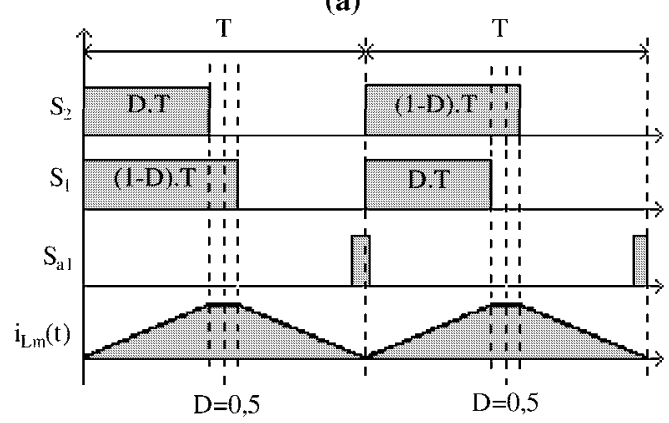

(b)

Figura 2 - Comando dos interruptores e corrente de magnetização do transformador: (a) Comando convencional. (b) Comando proposto.
Observa-se que com o uso do comando proposto (Fig. 2b), não se modifica a relação de transferência de potência do conversor forward com dois interruptores com comando convencional (Fig. 2a), pois somente se atrasa o momento do início da desmagnetização do transformador.

A seguir são apresentadas as nove etapas de funcionamento que ocorrem no conversor forward, ao se utilizar o comando e o circuito auxiliar proposto. Na análise que segue, todos os componentes são considerados ideais; 0 transformador é composto por indutância de dispersão e magnetizante, e o filtro de saída e a carga são substituídos por uma fonte de corrente ideal. A indutância de dispersão pode ser considerada tanto no circuito primário como no secundário, sem alterar a análise. Os circuitos equivalentes das etapas são apresentados na Fig. 3.

Etapa $1\left(\mathrm{t}_{0}-\mathrm{t}_{1}\right)$ : Considera-se inicialmente que a corrente no indutor $L_{d}$ é igual à corrente de carga. Os interruptores $S_{1}$ e $\mathrm{S}_{2}$ estão em condução e a fonte de tensão $\mathrm{E}$ transfere energia para a carga através de $S_{1}, T_{1}\left(N_{1}\right)$ e $S_{2}$ no primário, e $T_{1}\left(N_{2}\right)$, $L_{d}$ e $D_{3}$ no secundário. $O$ interruptor $S_{a 1}$ está bloqueado. $A$ corrente magnetizante do transformador, $\mathrm{i}_{\mathrm{Lm}}(\mathrm{t})$, aumenta linearmente, excitada pela fonte de tensão $\mathrm{E}$. A corrente em $\mathrm{T}_{1}(\mathrm{~N} 1), \mathrm{i}_{\mathrm{T} l(\mathrm{~N})}(\mathrm{t})$, é a soma das correntes de carga $\mathrm{I}_{\mathrm{c}}$ refletida para o primário e a corrente magnetizante do transformador $i_{L m}(t)$, sendo estas grandezas definidas em (1) e (2).

$$
\begin{gathered}
\mathrm{i}_{\mathrm{Lm}}(\mathrm{t})=\frac{\mathrm{E}}{\mathrm{L}_{\mathrm{m}}} \cdot \mathrm{t}+\mathrm{I}_{\mathrm{Lm} 9}, \\
\mathrm{i}_{\mathrm{T} 1(\mathrm{~N} 1)}(\mathrm{t})=\frac{\mathrm{E}}{\mathrm{L}_{\mathrm{m}}} \cdot \mathrm{t}+\mathrm{I}_{\mathrm{Lm} 9}+\mathrm{I}_{\mathrm{c}},
\end{gathered}
$$

sendo $I_{L m x}$ a corrente magnetizante no instante $t_{x}$, onde $x=1$, $2, \ldots, 9$.

Etapa $2\left(\mathrm{t}_{1}-\mathrm{t}_{2}\right)$ : Em $\mathrm{t}_{1}$, bloqueia-se somente o interruptor $\mathrm{S}_{2}$ (ZVS) e a tensão sobre o capacitor $\mathrm{C}_{2}$ começa a aumentar linearmente, devido à corrente de carga e à corrente magnetizante $\mathrm{i}_{\mathrm{Lm}}(\mathrm{t})$. A tensão no capacitor $\mathrm{C}_{2}$ e a corrente magnetizante são dadas por (3) e (4).

$$
\begin{gathered}
{ }^{v_{C} 2}(t)=E+Z_{1} \cdot\left(I_{c}+I_{L m 1}\right) \cdot \operatorname{sen}\left(\omega_{1} \cdot t\right), \\
i_{L m}(t)=\left(I_{c}+I_{L m 1}\right) \cdot \cos \left(\omega_{1} \cdot t\right)-I_{c},
\end{gathered}
$$

onde $Z_{1}$ é a impedância característica desta etapa e $\omega_{1}$ é a freqüência angular ressonante. Estas grandezas são definidas por:

$$
\begin{gathered}
\mathrm{Z}_{1}=\sqrt{\frac{\mathrm{L}_{\mathrm{m}}}{\mathrm{C}}}, \\
\omega_{1}=\frac{1}{\sqrt{\mathrm{L}_{\mathrm{m}} \cdot \mathrm{C}}},
\end{gathered}
$$

sendo: $\mathrm{C}=\mathrm{C}_{1}=\mathrm{C}_{2}$.

Etapa $3\left(\mathrm{t}_{2}-\mathrm{t}_{3}\right):$ Em t $\mathrm{t}_{2}$, a tensão sobre o capacitor $\mathrm{C}_{2}$ atinge a tensão $\mathrm{E}$ e o diodo $\mathrm{D}_{2}$ entrada em condução. Com isso, a corrente magnetizante $i_{\mathrm{Lm}}(t)$ e a corrente de carga $I_{c}$ ficam em roda livre através do diodo $\mathrm{D}_{2}$ e do interruptor $\mathrm{S}_{1}$.

Etapa $4\left(\mathrm{t}_{3}-\mathrm{t}_{4}\right)$ : No instante $\mathrm{t}_{3}$, o interruptor $\mathrm{S}_{1}$ é bloqueado (ZVS) e a corrente que antes circulava por este interruptor passa a circular através do capacitor $\mathrm{C}_{1}$. Ocorre a ressonância 


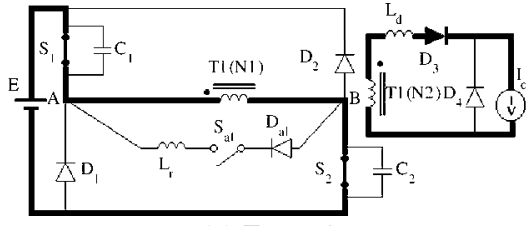

(a) Etapa 1

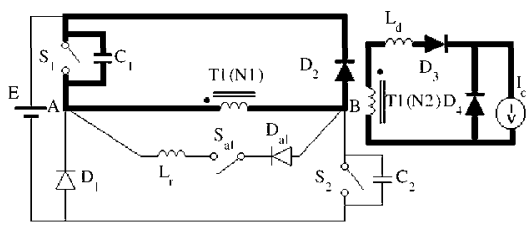

(d) Etapa 4

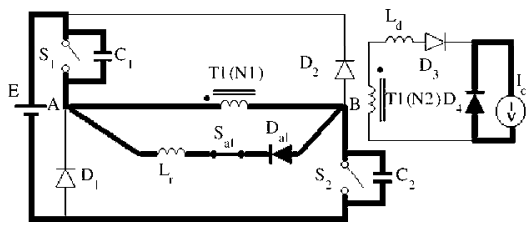

(g) Etapa 7

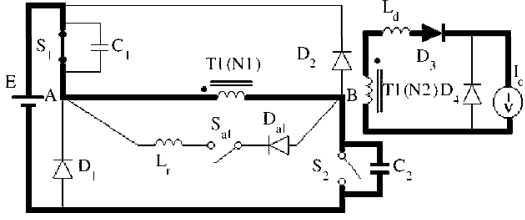

(b) Etapa 2

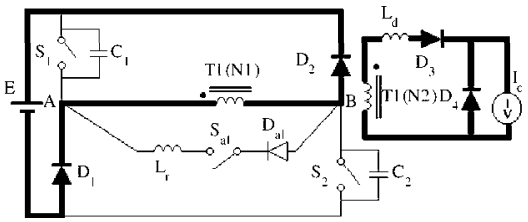

(e) Etapa 5

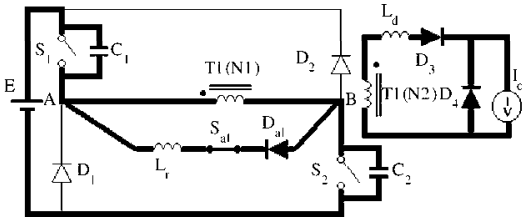

(h) Etapa 8

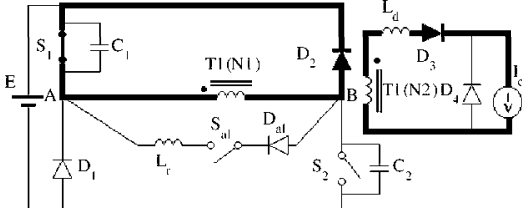

(c) Etapa 3

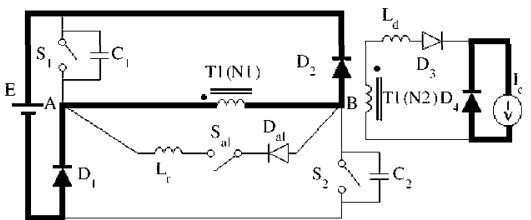

(f) Etapa 6

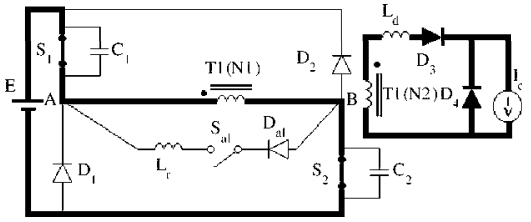

(i) Etapa 9

Figura 3 - Etapas de Operação.

entre $C_{1}, L_{m}$ e $L_{d}$, decrescendo a corrente em $L_{d}$ e $L_{m}$ e aumentando a tensão sobre o capacitor $C_{1}$. Neste período começa a haver a transferência da corrente do diodo $\mathrm{D}_{3}$ para o diodo $\mathrm{D}_{4}$, devido à inversão da tensão $\mathrm{V}_{\mathrm{AB}}$. As equações relevantes desta etapa são:

$$
\begin{gathered}
{ }_{\mathrm{Cl}}(\mathrm{t})=\mathrm{Z}_{2} \cdot\left(\mathrm{I}_{\mathrm{Lm} 3}+\mathrm{I}_{\mathrm{c}}\right) \cdot \operatorname{sen}\left(\omega_{2} \cdot \mathrm{t}\right), \\
{ }_{\mathrm{Lm}}(\mathrm{t})=\mathrm{I}_{\mathrm{Lm} 3}-\mathrm{A}_{1} \cdot\left(\mathrm{I}_{\mathrm{Lm} 3}+\mathrm{I}_{\mathrm{c}}\right) \cdot\left(1-\cos \left(\omega_{2} \cdot \mathrm{t}\right),\right. \\
\mathrm{i}_{\mathrm{Ld}}(\mathrm{t})=\left(\mathrm{I}_{\mathrm{Lm} 3}+\mathrm{I}_{\mathrm{c}}\right) \cdot\left(\mathrm{A}_{1}+\cos \left(\omega_{2} \cdot \mathrm{t}\right) \cdot\left(1-\mathrm{A}_{1}\right)-\mathrm{I}_{\mathrm{Lm} 3},\right.
\end{gathered}
$$

Onde: $A_{1}$ é uma constante, e $Z_{2}$ e $\omega_{2}$ são a impedância característica e a freqüência angular respectivamente.

$$
\begin{aligned}
& A_{1}=\frac{L_{d}}{L_{d}+L_{m}}, \\
& Z_{2}=\sqrt{\frac{L_{\text {eql }}}{C}}, \\
& \omega_{2}=\frac{1}{\sqrt{L_{\text {eq1 }} \cdot C}} .
\end{aligned}
$$

Onde:

$$
\mathrm{L}_{\mathrm{eql}}=\frac{\mathrm{L}_{\mathrm{d}} \cdot \mathrm{L}_{\mathrm{m}}}{\mathrm{L}_{\mathrm{d}}+\mathrm{L}_{\mathrm{m}}} .
$$

Etapa $5\left(\mathrm{t}_{4}-\mathrm{t}_{5}\right)$ : No instante $\mathrm{t}_{4}$, a tensão sobre o capacitor $\mathrm{C}_{1}$ atinge a tensão $\mathrm{E}$ e o diodo $\mathrm{D}_{1}$ entra em condução. Aplica-se -E Volts entre os pontos A e B e a corrente magnetizante $\mathrm{i}_{\mathrm{Lm}}(\mathrm{t})$ que já decrescia de forma ressonante, agora começa a decrescer linearmente. No secundário, a corrente no indutor $\mathrm{L}_{\mathrm{d}}$ também decresce linearmente. As correntes são definidas por:

$$
\mathrm{i}_{\mathrm{Lm}}(\mathrm{t})=\mathrm{I}_{\mathrm{Lm} 4}-\frac{\mathrm{E}}{\mathrm{L}_{\mathrm{m}}} \cdot \mathrm{t},
$$

$$
\begin{gathered}
\mathrm{i}_{\mathrm{Lm}}(\mathrm{t})=\mathrm{I}_{\mathrm{Lm} 4}-\frac{\mathrm{E}}{\mathrm{L}_{\mathrm{m}}} \cdot \mathrm{t}, \\
\mathrm{i}_{\mathrm{Ld}}(\mathrm{t})=\mathrm{I}_{\mathrm{Ld} 4}-\frac{\mathrm{E}}{\mathrm{L}_{\mathrm{d}}} \cdot \mathrm{t}, \\
\mathrm{i}_{\mathrm{T} 1}(\mathrm{t})=\mathrm{I}_{\mathrm{Lm} 4}+\mathrm{I}_{\mathrm{Ld} 4}-\frac{\mathrm{E}}{\mathrm{L}_{\text {eql }}} \cdot \mathrm{t} .
\end{gathered}
$$

Etapa $6\left(\mathrm{t}_{5}-\mathrm{t}_{6}\right)$ : No instante $\mathrm{t}_{5}$, a corrente no indutor $\mathrm{L}_{\mathrm{d}}$ tornase nula, o diodo $\mathrm{D}_{3}$ bloqueia e a corrente de carga passa a circular pelo diodo $\mathrm{D}_{4}$. A corrente magnetizante é definida por:

$$
\mathrm{i}_{\mathrm{Lm}}(\mathrm{t})=\mathrm{I}_{\mathrm{Lm} 5}-\frac{\mathrm{E}}{\mathrm{L}_{\mathrm{m}}} \cdot \mathrm{t} .
$$

Etapa $7\left(\mathrm{t}_{6}-\mathrm{t}_{7}\right)$ : No instante $\mathrm{t}_{6}$, a corrente magnetizante $\mathrm{i}_{\mathrm{Lm}}(\mathrm{t})$ torna-se nula. Neste instante, $S_{\mathrm{al}}$ é comandado para a condução de forma ZCS devido à presença de $\mathrm{L}_{\mathrm{r}}$. Dá-se inicio à ressonância entre a energia dos capacitores $C_{1}$ e $C_{2} \mathrm{e}$ a energia dos indutores $L_{r}$ e $L_{m}$, provocando o inicio da inversão da tensão $v_{A B}$. Como $L_{m}>L_{r}$, a indutância de magnetização pode ser desconsiderada para efeito de projeto. A tensão nos capacitores e a corrente no indutor ressonante são dados por:

$$
\begin{gathered}
v_{C}(t)=v_{C 1}(t)=v_{C \cdot 2}(t)=E \cdot \cos \left(\omega_{3} \cdot t\right)+E \\
i_{L r}(t)=-\frac{E}{Z_{3}} \cdot \operatorname{sen}\left(\omega_{3} \cdot t\right)
\end{gathered}
$$

onde $Z_{3}$ é a impedância característica e $\omega_{3}$ é a frequiência angular ressonante definidas em (20) e (21).

$$
\begin{gathered}
\mathrm{Z}_{3}=\sqrt{\frac{\mathrm{L}_{\mathrm{r}}}{\mathrm{C}_{\mathrm{eq}}}}, \\
\omega_{3}=\frac{1}{\sqrt{\mathrm{L}_{\mathrm{r}} \cdot \mathrm{C}_{\mathrm{eq}}}},
\end{gathered}
$$


sendo $\mathrm{C}_{\mathrm{eq}}$ definido em (22):

$$
\mathrm{C}_{\mathrm{eq}}=\frac{\mathrm{C}}{2} \text {. }
$$

Etapa $8\left(\mathbf{t}_{7}-\mathrm{t}_{8}\right)$ : No instante $\mathrm{t}_{7}$ as tensões sobre os capacitores $\mathrm{C}_{1}$ e $\mathrm{C}_{2}$ atingem $\mathrm{E} / 2$ Volts. A partir deste instante é aplicada novamente tensão positiva entre os pontos $\mathrm{AB}$, permitindo a entrada em condução do diodo $\mathrm{D}_{3}$. Há, então, a ressonância entre a energia dos capacitores $\mathrm{C}_{1}$ e $\mathrm{C}_{2}$ com a dos indutores $\mathrm{L}_{\mathrm{r}}$ e $\mathrm{L}_{\mathrm{d}}$. A indutância magnetizante é desconsiderada, pois $\mathrm{L}_{\mathrm{m}} \gg \mathrm{L}_{\mathrm{l}}$. As principais grandezas relacionadas para esta etapa são:

$$
\begin{gathered}
{ }^{v_{C}}(t)=\mathrm{L}+Z_{4} \cdot I_{L r} \cdot \operatorname{sen}\left(\omega_{4} \cdot t\right) \\
i_{L r}(t)=I_{L r}-A_{2} \cdot I_{L r} \cdot\left[1-\cos \left(\omega_{4} \cdot t\right)\right], \\
i_{L d}(t)=I_{L r 7} \cdot \cos \left(\omega_{4} \cdot t\right)+A_{2} \cdot I_{L r 7} \cdot\left[1-\cos \left(\omega_{4} \cdot t\right)\right]-I_{L r 7},
\end{gathered}
$$

onde $I_{L r 7} \hat{c}$ a corrente no indutor ressonante $L_{r}$ no instante $t_{7}$, $\mathrm{Z}_{4}$ a impedância característica desta etapa, $\omega_{4}$ é frequiência angular do circuito ressonante $\mathrm{e}_{2}$ é uma constante, definidas como:

$$
\begin{gathered}
\mathrm{A}_{2}=\frac{\mathrm{L}_{\mathrm{d}}}{\mathrm{L}_{\mathrm{d}}+\mathrm{L}_{\mathrm{r}}}, \\
\mathrm{Z}_{4}=\sqrt{\frac{\mathrm{L}_{\mathrm{eq} 2}}{\mathrm{C}_{\mathrm{eq}}}} \\
\omega_{4}=\frac{1}{\sqrt{\mathrm{L}_{\mathrm{eq} 2} \cdot \mathrm{C}_{\mathrm{eq}}}},
\end{gathered}
$$

Onde:

$$
L_{e q 2}=\frac{L_{d} \cdot L_{r}}{L_{d}+L_{r}}
$$

Etapa $9\left(\mathbf{t}_{8}-\mathrm{t}_{9}\right)$ : Em $\mathrm{t}_{8}$ as tensões sobre os capacitores $\mathrm{C}_{1} \mathrm{e}_{2}$ atingem tensão próxima de zero Volt e os interruptores $S_{1}$ e $\mathrm{S}_{2}$ são comandados para a condução de forma quasi-ZVS, aplicando a fonte de tensão E sobre os terminais do primário de $\mathrm{T}_{1}(\mathrm{~N} 1)$. Havendo corrente nula em $\mathrm{L}_{\mathrm{l}}, \mathrm{S}_{\mathrm{a} 1}$ é bloqueado de forma ZCS. A corrente no indutor $\mathrm{L}_{\mathrm{d}}$ aumenta linearmente, permitindo a transferência da corrente de carga do diodo $\mathrm{D}_{4}$ para o diodo $\mathrm{D}_{3}$. No instante $\mathrm{t}_{9}$, a corrente no indutor $\mathrm{L}_{\mathrm{d}}$ atinge o valor da corrente de carga $I_{c}$. As correntes nesta etapa são:

$$
\begin{gathered}
\mathrm{i}_{\mathrm{Lm}}(\mathrm{t})=\frac{\mathrm{E}}{\mathrm{L}_{\mathrm{m}}} \cdot \mathrm{t}+\mathrm{I}_{\mathrm{Lm} 8}, \\
\mathrm{i}_{\mathrm{Ld}}(\mathrm{t})=\frac{\mathrm{E}}{\mathrm{L}_{\mathrm{d}}} \cdot \mathrm{t}+\mathrm{I}_{\mathrm{Ld} 8}, \\
\mathrm{i}_{\mathrm{T} 1(\mathrm{~N} 1)}(\mathrm{t})=\frac{\mathrm{E}}{\mathrm{L}_{\mathrm{eq} 2}} \cdot \mathrm{t}+\mathrm{I}_{\mathrm{Lm} 8}+\mathrm{I}_{\mathrm{Ld} 8} \cdot
\end{gathered}
$$

Na Fig. 4 são apresentadas as principais formas de onda teóricas para o conversor forward com dois interruptores, utilizando a lógica de comando proposta.

Ao projetar o conversor forward, deve-se ter uma atenção especial com a corrente magnetizante do transformador. Nas etapas 2 e 4, onde as comutações ocorrem dos interruptores para os diodos, a corrente de carga exerce forte influência. Se o conversor operar sem carga ou com baixo valor de corrente de carga, haverá um aumento no tempo de comutação, resultando no aumento da razão cíclica efetiva. Desta forma, uma compensação na razão cíclica deve ser feita para evitar que a corrente magnetizante adquira um valor $\mathrm{CC}$.

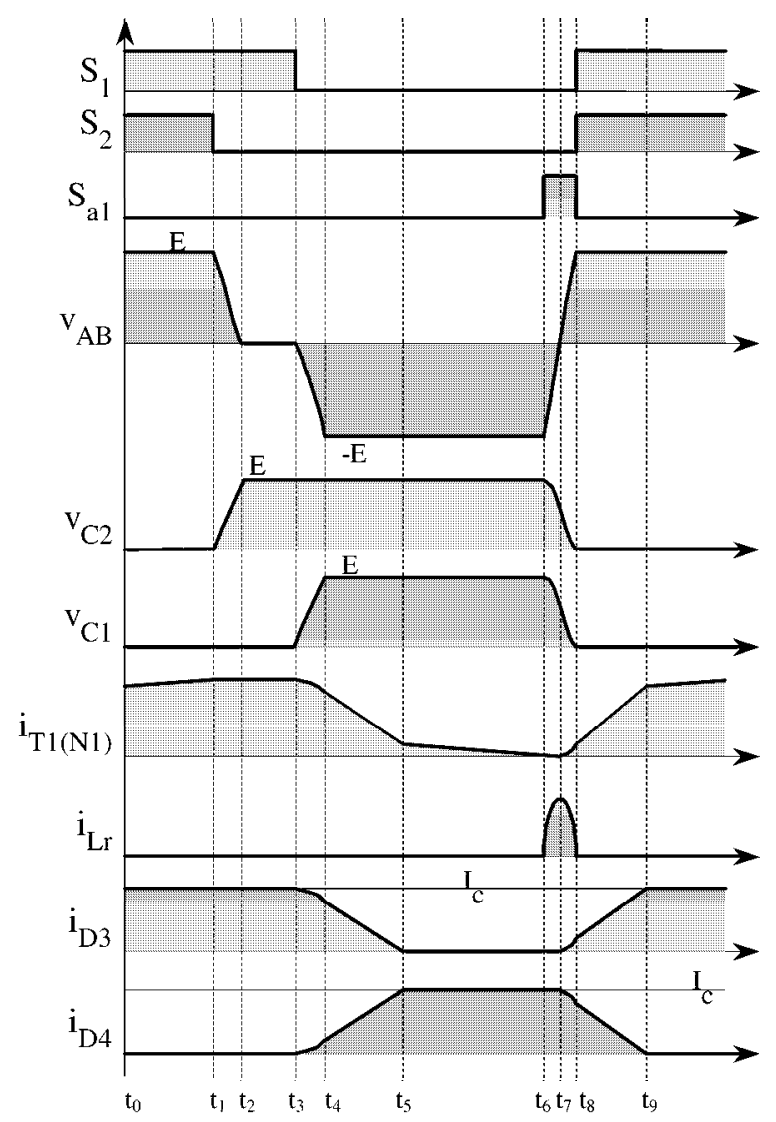

Figura 4 - Principais formas de onda teóricas

\section{Procedimentos DE PRoJeto:}

O principal cuidado ao se projetar o indutor ressonante é garantir que na etapa 8 , quando houver a ressonância entre $\mathrm{C}_{1}, \mathrm{C}_{2}, \mathrm{~L}_{\mathrm{r}}$ e $\mathrm{L}_{\mathrm{d}}$, a tensão sobre os capacitores atinja um valor próximo de zero. Esta é a característica do quasi-ZVS proposto.

No momento em que a tensão cntre os pontos $A D$ atinge zero Volt $\left(\mathrm{V}_{\mathrm{AB}}=0\right)$ em $\mathrm{t}=\mathrm{t}_{7}$, inicia-se a transferência da corrente de carga do diodo $\mathrm{D}_{4}$ para o diodo $\mathrm{D}_{3}$. O indutor $\mathrm{L}_{\mathrm{d}}$ passa a fazer parte da ressonância, conforme pode ser melhor visualizado no circuito equivalente representado na Fig. 5. $\mathrm{L}_{\mathrm{m}}$ é desprezada nesta etapa, uma vez que $\mathrm{L}_{\mathrm{r}}$ é muito menor do que a indutância magnetizante do transformador.

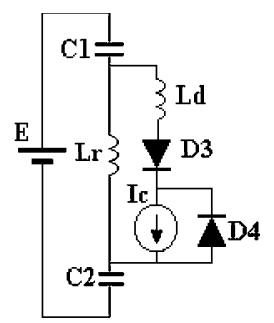

Figura 5 - Circuito ressonante equivalente da etapa 8

No circuito da Fig. 5, as energias nos capacitores $C_{1}$ e $C_{2}$ ressonam com aquela do indutor equivalente aos indutores $\mathrm{L}_{r}$ e $L_{d}$ em paralelo. Nesta ressonância, define-se um valor de 
tensão próximo de zero ( $\left.\mathrm{V}_{\mathrm{QzvS}}\right)$, no qual os interruptores principais devem entrar em condução. Assim, o indutor ressonante pode ser calculado a partir da equação (33).

$$
\mathrm{L}_{\mathrm{r}} \leq \frac{\mathrm{L}_{\mathrm{d}} \cdot\left[\mathrm{E}^{2}-\left(\mathrm{E}-\mathrm{V}_{\mathrm{QZVS}}\right)^{2}\right\rfloor}{\left(\mathrm{E}-\mathrm{V}_{\mathrm{QZVS}}\right)^{2}}
$$

\section{EXEMPLO DE PROJETO}

A seguir é apresentado um exemplo de projeto para o conversor proposto:

Tensão de entrada: $\mathrm{E}=200 \mathrm{~V}$

Potência de saída: $\mathrm{Po}=250 \mathrm{~W}$

Tensão de saída: $\mathrm{V}_{0}=48 \mathrm{~V}$

Frequência de chaveamento: $\mathbf{f}_{\mathrm{s}}=100 \mathrm{kHz}$

Razão cíclica: $\mathrm{D}=0,45$

Relação de transformação: $n=\frac{N_{1}}{N_{2}}=1,8 / 1$

Indutância de dispersão medida: $\mathrm{L}_{\mathrm{d}}=18 \mu \mathrm{H}$

Definindo $\mathrm{V}_{\mathrm{QZVS}}=20 \mathrm{~V}$,

Então:

$\mathrm{L}_{\mathrm{r}} \leq 4,22 \mu \mathrm{H}$

O valor de $4,22 \mu \mathrm{H}$ é o valor máximo da indutância de $\mathrm{L}_{\mathrm{s}}$ para que os interruptores principais comutem com $20 \mathrm{~V}$, na

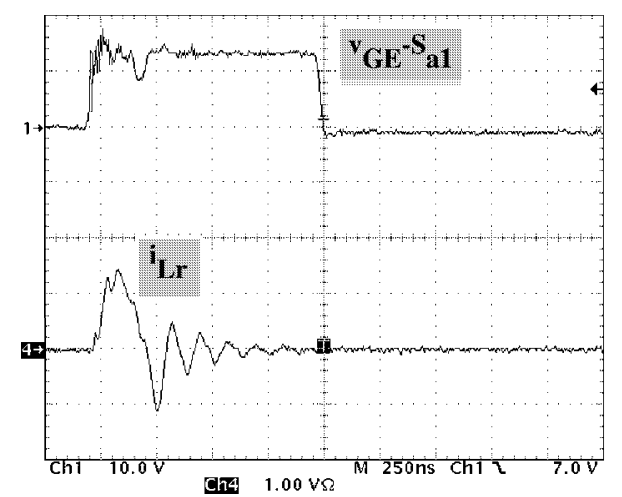

(a)

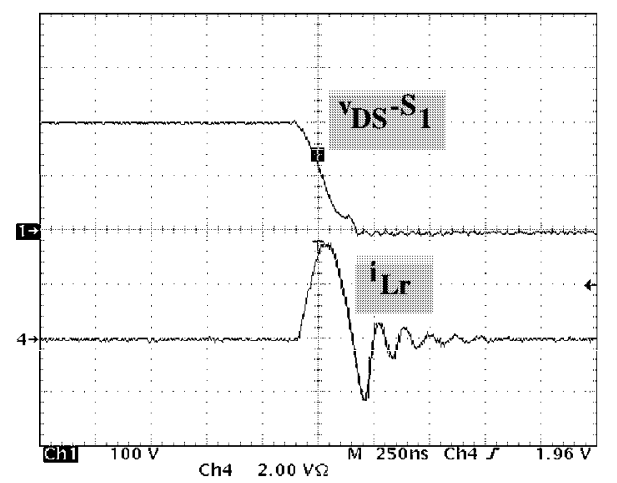

(c) entrada em condução, considerando que o circuito auxiliar tenha um alto fator de qualidade.

\section{RESULTADOS EXPERIMENTAIS}

Para a implementação da topologia proposta, fora utilizados os parâmetros do exemplo de projeto. A Tabela I apresenta a lista dos dispositivos e componentes que foram utilizados na implementação do protótipo.

Tabela I

Dispositivos e componentes

\begin{tabular}{ll} 
Componentes & Especificações \\
\hline $\mathrm{S}_{1}, \mathrm{~S}_{2}$ & MOSFETs IRFP450 \\
$\mathrm{S}_{21}$ & IGBT G3N60C3D \\
$\mathrm{D}_{1}, \mathrm{D}_{2}$ & RHRP870 \\
$\mathrm{D}_{3}, \mathrm{D}_{4}$ & MUR1515 \\
$\mathrm{D}_{21}$ & HFA25PB60 \\
$\mathrm{Tr}_{\mathrm{n}}$ sformador $\mathrm{T}_{1},(\mathrm{~N} 1, \mathrm{~N} 2)$ & EE-65/26, $\left(\mathrm{N}_{1}: \mathrm{N}_{2}\right)=22: 14$ Thornton \\
$\mathrm{Lr}$ & EE-30/14 -5 espiras $=3,7 \mathrm{uH}$ Thornton \\
$\mathrm{C}_{1}, \mathrm{C}_{2}$ & $2,2 \mathrm{n}$
\end{tabular}

Para a implementação dos sinais de comando para os interruptores foi usado um Dispositivo Lógico Programável Apagável - EPLD (EPM7128LC84-7/ALTERA) operando com frequiência de $33,33 \mathrm{MHz}$ em malha aberta. Com este ASIC é possível fazer o período de desmagnetização adequado para controlar o fluxo magnetizante, usando programação lógica digital AHDL (Altera Hardware Descrition Language).

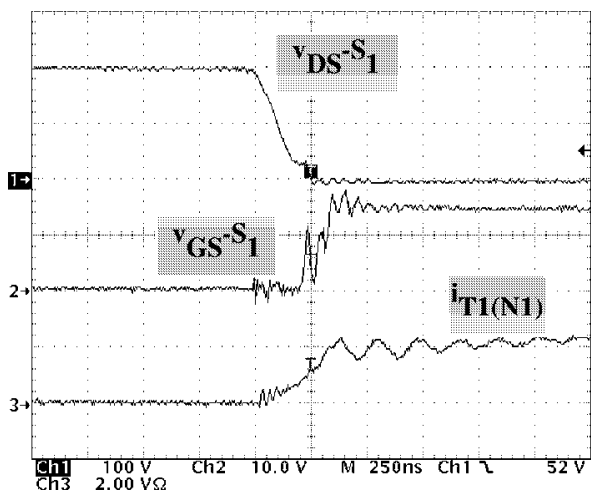

(b)

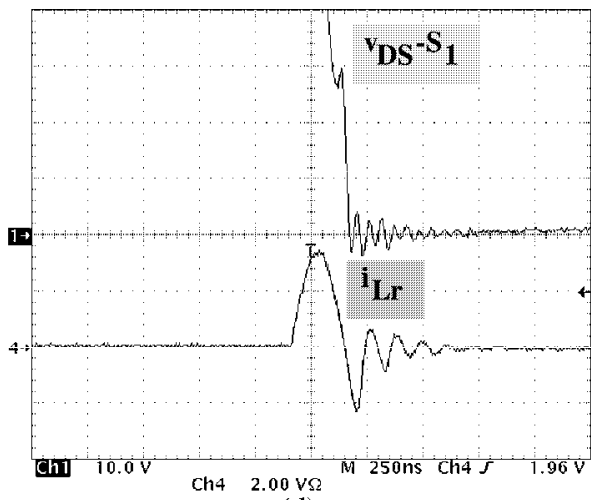

(d)

Figura 6 - Formas de Ondas Experimentais das Comutações Suaves: (a) Tensão gatilho-emissor e corrente coletor-emisor no interruptor $S_{a 1}$, (b) Tensão dreno-fonte e tensão gatilho-fonte no interruptor $S_{1}$ e corrente no primário do transformador. (c) Tensão dreno-fonte no interruptor $S_{1}$ e corrente no indutor ressonante, (d) detalhe da tensão dreno-fonte no interruptor $S_{1}$ e corrente no indutor ressonante. 
Esta EPLD tornou o circuito de comando compacto, simples e flexível. Além disto, durante o estágio de prototipagem, a implementação deste dispositivo programável tornou-se muito adcquado, pois qualquer modificação no circuito de comando é feita rapidamente pelo software, mantendo-se o hardware inalterado.

$\mathrm{Na}$ Fig. 6a são apresentados o sinal de gatilho do interruptor Sa1 e sua corrente coletor-emissor, demonstrando a comutação ZCS deste interruptor.

A comutação quasi-ZVS do interruptor principal $S_{1}$ pode ser vista na Fig. 6b. Nesta figura, pode-se ver que o sinal de gatilho é aplicado quando a tensão sobre o interruptor principal está próxima de zero Volt, no final da oitava etapa. Ainda nesta figura, é apresentada a corrente no primário do transformador $(\mathrm{T} 1(\mathrm{~N} 1)$ ), demonstrando que a corrente só aumenta efetivamente após a tensão atingir $100 \mathrm{~V}$ (metade do valor da tensão da fonte). Neste instante, a tensão entre A e $\mathrm{B}$ torna-se positiva.

A ressonância entre a energia no capacitor $C_{1}$ e no indutor Lr é mostrada na Fig. 6c. A Fig. 6d apresenta a mesma forma de onda, mas com a escala vertical ampliada para melhor visualização da comutação quasi-ZVS. Nesta figura pode-se ver o nível de tensão de comutação quasi-ZVS ( $\mathrm{V}_{\mathrm{QZVS}}$ aproximadamente $26 \mathrm{~V}$ ).

Uma das vantagens do circuito auxiliar à comutação é que ele apresenta baixo valor de corrente eficaz e média. Isto pode ser visto na Fig. 7a, onde estão apresentadas as correntes do primário do transformador e aquela através de $\mathrm{L}_{\mathrm{r}}$.
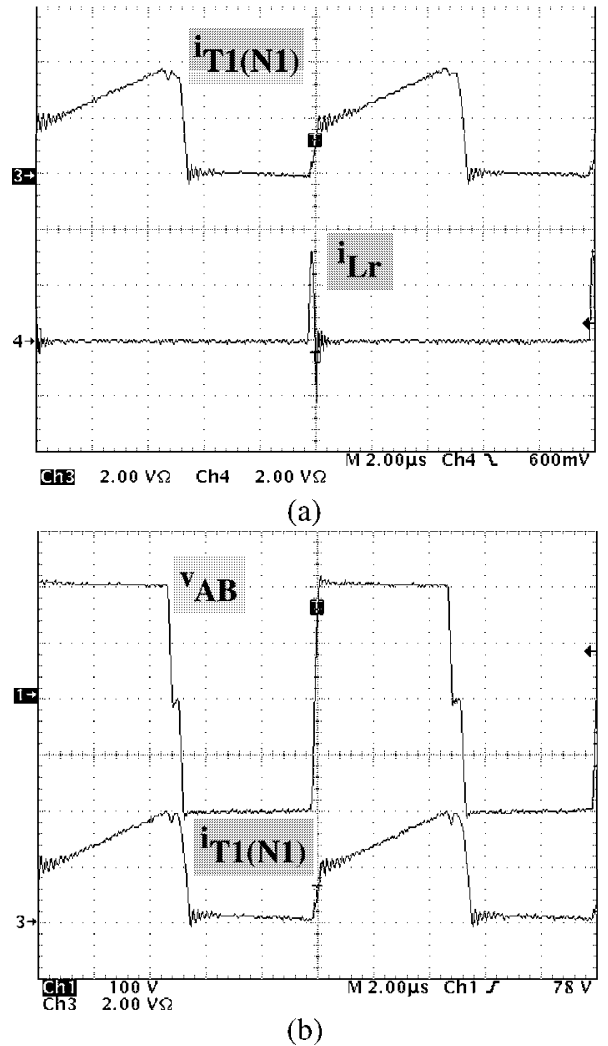

Figura 7 - Formas de Ondas Experimentais:

(a) Corrente no primário do transformador e corrente no indutor ressonante. (b) Tensão e corrente no primário do transformador.
A Fig. 7b apresenta a tensão e a corrente no primário do transformador, onde se pode verificar os três níveis em $\mathrm{V}_{\mathrm{AB}}$.

As curvas dos rendimentos, para comparação entre o conversor Quasi-ZVS proposto e o conversor hard estão mostradas na Fig. 8. Nesta figura pode-se verificar o considerável aumento de eficiência do conversor Quasi-ZVS (91\%) em relação ao conversor hard $(86,5 \%)$ a plena carga.

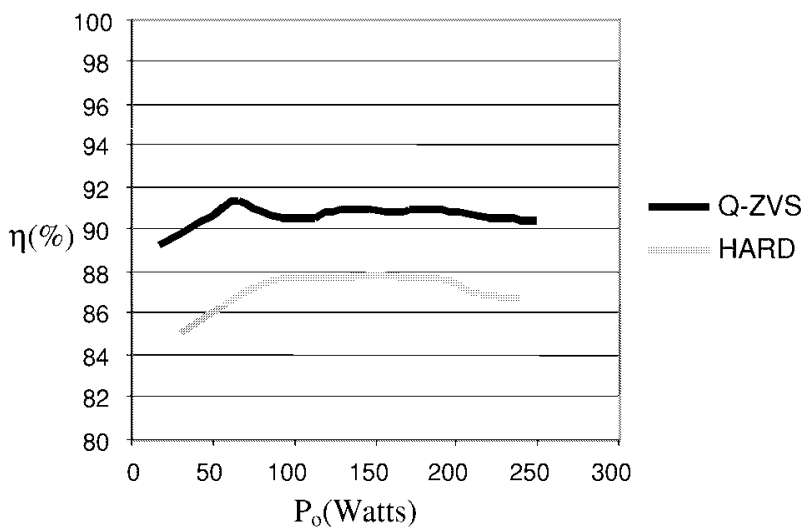

Figura 8 - Curvas dos Rendimentos para Conversor Hard e o Quasi-ZVS Proposto.

\section{CONCLUSÕES}

Neste artigo é apresentado e analisado um circuito de auxilio à comutação e um comando lógico aplicado ao conversor forward com dois interruptores. Além disto, é introduzido um novo conceito de comutação, onde é permitido e projetado que os interruptores principais comutem em um modo Quasi-ZVS na entrada em condução.

O circuito adicional auxiliar apresenta poucos componentes e um valor de corrente eficaz e médio muito baixo, que resulta em um reduzido custo e volume. Neste circuito auxiliar os dispositivos semicondutores comutam sob zero de corrente, caracterizando-se como um circuito de baixas perdas de comutação.

$\mathrm{O}$ indutor $\mathrm{L}_{\mathrm{d}}$ é a própria indutância de dispersão do transformador, não sendo necessário a inclusão de u indutor externo adicional.

O elevado rendimento é obtido devido ao fato do conversor operar com baixas perdas de comutação e com razão cíclica próxima de $50 \%$. O protótipo implementado obteve um rendimento de $91 \%$ a plena carga.

\section{REFERÊNCIAS BIBLIOGRÁFICAS}

[1] A.A. Pereira, E. Coelho, V.Farias, L. Freitas, J.B. Júnior; "A New ZC-ZVS Forward Converter"; APEC'96 Record, pp. $482-486$.

[2] C. Treviso, A. Pereira, V. Farias, J.B. Vierira Jr., L. Freitas; " A $1.5 \mathrm{~kW}$ Operation with $90 \%$ Efficiency of a Two Transistors Forward Converter with non-dissipative snubber; PESC'98 Record, pp. 696-700. 
[3] L. Freitas, A. Pereira, J. Andres, " A High Power Factor Operating Self-Resonant - PWM Forward Converter", APEC'95 Record, pp. 440-446.

[4] C. Dias, A. Pereira, J.B. Vierira Jr, V. Farias, L. Freitas; "An Improved Self-Resonant PWM Forward Converter, APEC'98, Record, pp. 620-625.

[5] N.H.Kutkut, G. Luckjiff, D.M. Divan; "A Dual High Current DC-To-DC Converter With Soft Switching Capability", IAS'97 Record, pp. 1398-1405.

[6] Vidor, D.L.R.; Pinheiro, H.; Vasconcelos, M. A; Fagundes, R.F.; "Estudo de um Conversor PWM CCCC ou CC-CA Quatro Quadrantes com Baixas perdas nas Comutações", III Seminário de Eletrônica de Potência, 1990, pp. 186-193.

\section{DADOS BIOGRÁFICOS}

José Renes Pinheiro nasceu em Santa Maria - RS, em 1958. Formou-se em Engenharia Elétrica pela Universidade Federal de Santa Maria, em 1981. Obteve o título de Mestre em Engenharia Elétrica pela Universidade Federal de Santa
Catarina, em 1984 e o título de Doutor em Engenharia na mesma universidade, em 1994. Atualmente, é professor Titular do departamento de Eletrônica e Computação da Universidade Federal de Santa Maria. É membro da SOBRAEP, SBA e IEEE-PELS-IES-IAS-CS. Suas áreas de interesse compreendem Técnicas de Comutação Suave, PréReguladores, Filtros e Controle de Conversores Estáticos.

José Eduardo Baggio nasceu em Santa Maria - RS, em 1971. Formou-se em Engenharia Elétrica pela Universidade Federal de Santa Maria, em 1995. Obteve o título de Mestre em Engenharia Elétrica na Universidade Federal de Santa Maria, em 1997. Atualmente é estudante de doutorado na mesma universidade. Sua área de interesse compreende Eletrônica de Potência, Sistemas de Controle e Sistemas Digitais.

Luciano Schuch nasceu em Santa Maria - RS, em 1974. Formou-se em Engenharia Elétrica pela Universidade Federal de Santa Maria, em 1999. Atualmente é estudante de mestrado na mesma universidade. Sua área de interesse compreende Eletrônica de Potência e Sistemas Digitais. 